\title{
Awareness, consecuencias de una experiencia estresante
}

\author{
VÍCTOR LUENGO J. ${ }^{1}$, CAROLA ZAPATA P. ${ }^{1}$, ALEJANDRO DELFINO ${ }^{2}$, \\ JORGE CALDERÓN ${ }^{1}$, MATÍAS GONZÁLEZ ${ }^{1}$
}

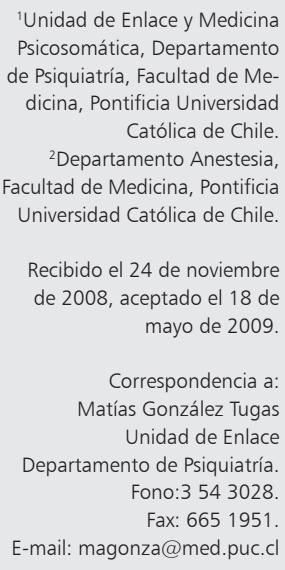

'Unidad de Enlace y Medicina Psicosomática, Departamento de Psiquiatría, Facultad de Medicina, Pontificia Universidad Católica de Chile. 2Departamento Anestesia Facultad de Medicina, Pontificia Universidad Católica de Chile.

Recibido el 24 de noviembre de 2008, aceptado el 18 de mayo de 2009.

Correspondencia a: Matías González Tugas Unidad de Enlace Departamento de Psiquiatría. Fono:3 543028 Fax: 6651951 E-mail: magonza@med.puc.cl

\begin{abstract}
Anesthesia awareness
Anesthesia awareness, or "unintended intra-operative awareness" occurs during general anesthesia, on the operating table, when a patient has not had enough general anesthetic or analgesic to prevent consciousness or waking up during surgery. According to international studies its global incidence ranges from 0.1 to $0.2 \%$. Its impact on people can be as severe as other traumatic experiences such as natural disasters, violent fights or sexual abuse. The prevalence of symptoms compatible with post traumatic stress disorder can be as high as 50\%, after experiencing the awareness phenomenon. This paper reviews the main issues of the awareness phenomenon.
\end{abstract}

(Rev Med Chile 2010; 138: 352-357).

Key words: Anesthesia, conduction; Stress disorders, post-traumatic; Awareness.
$\mathrm{C}$ onstituyen pilares fundamentales de toda anestesia general la inconsciencia, amnesia, analgesia, relajación neuromuscular y estabilidad autonómica. Precisamente, el fenómeno del awareness se correlaciona estrechamente con la inconsciencia y la amnesia y en menor medida con la analgesia. Otro parámetro relevante e íntimamente relacionado con el awareness es la memoria, la que puede ser dividida operacionalmente en dos categorías ${ }^{1,2}$ :

a) Memoria explícita o consciente: que consiste en la recuperación intencionada de información previa; b) Memoria implícita o inconsciente: recolección inconsciente de experiencias previas.

Estamos en presencia de awareness cuando se produce la adquisición o formación de memoria explícita durante la anestesia general ${ }^{3}$. Sin embargo, la memoria implícita también parece influir en el comportamiento posterior, ya que al no ser manejado por la consciencia podría generar un mayor impacto emocional. No está claro aún si la activación de los diferentes tipos de memoria se produce de forma independiente o son fases de un mecanismo continuo.

Los factores que pueden contribuir al desarrollo del awareness son múltiples, sin embargo, todos ellos traen como consecuencia un inadecuado plano de profundidad anestésica, entendido éste como el nivel de hipnosis durante la anestesia general, que condiciona el desarrollo de recuerdos intraoperatorios.

El nivel de profundidad anestésica es una tarea difícil de precisar y muchas veces subjetiva, en que el anestesiólogo debe ser capaz de interpretar signos clínicos inmersos en un proceso dinámico, condicionado tanto por los efectos depresores de los fármacos como por los estímulos nociceptivos. Algunos signos clínicos utilizados para esto son la abolición del reflejo palpebral y la ausencia de respuesta a la orden verbal, luego, durante el procedimiento, se suele utilizar la ausencia de movimientos y la monitorización hemodinámica, todos ellos predictores de baja correlación con el nivel de profundidad anestésica real. Dado lo anterior, desde hace algunos años existen en el mercado diversos monitores de profundidad anestésica y que se han utilizado con el fin de realizar una monitorización intraoperatoria más efectiva. En nuestro país existen pocos centros que cuentan con este recurso, siendo el Índice Bi Espectral (BIS) el monitor más difundido. Otros monitores existentes, aunque muy escasos, son el CSM (Cerebral 
State monitor) y el monitor de potenciales evocados auditivos. Todos ellos procesan una señal de EEG mediante un algoritmo particular para cada uno y esto se traduce en la entrega de un número que se correlaciona con el grado de profundidad anestésica, sin embargo, todos, en mayor o menor medida, presentan una serie de problemas técnicos y metodológicos que hacen que en la actualidad su interpretación no siempre corresponda con la clínica, lo que dificulta la generalización de su uso ${ }^{4}$.

La dificultad de obtener un dato objetivo y preciso en el nivel de profundidad anestésica puede provocar tanto una sobredosificación de fármacos, ocasionando desde retardo en los tiempos de despertar y de descarga de recuperación, con las consiguientes implicancias económicas que esto trae consigo, llegando incluso a existir trabajos que sugieren que una mayor profundidad anestésica puede ir asociada a una mayor mortalidad ${ }^{5}$, como la administración de dosis subterapéuticas, lo que puede asociarse al desarrollo de awareness y éste, a su vez, el desarrollo de trastorno de estrés post traumático ${ }^{6-8}$.

\section{Epidemiología}

Se estima que el fenómeno de awareness presenta una incidencia global de 0,1 a $0,2 \%$, llegando hasta $1 \%$ en algunas series. Si lo llevamos a números totales, tenemos que en Estados Unidos de Norteamérica, donde se dispone de la información epidemiológica más completa, ocurren entre 20.000 a 40.000 episodios al año. En grupos seleccionados, como cirugía cardíaca o trauma, estas cifras se elevan hasta 1,5 y 11 a $43 \%$ respectivamente $^{9-12}$. La dificultad de realizar estudios prospectivos y la baja incidencia de awareness han hecho difícil su estudio ${ }^{13}$. Sin embargo, se considera que desde la década 1960-69 ha disminuido su prevalencia, principalmente por el reconocimiento del fenómeno como algo real por parte de la comunidad médica, lo que conlleva una mejora en la prevención y por la irrupción de nuevos fármacos y técnicas que permiten evaluar de mejor manera el nivel de profundidad de la anestesia ${ }^{14,15}$.

\section{Detección}

Para la detección de awareness, el método validado es el cuestionario de Brice ${ }^{16}$, que consiste en cuatro preguntas que son: ¿qué es lo último que recuerda antes de dormir?; ¿qué es lo primero que recuerda al despertar?; ¿ recuerda algo entre el momento de dormirse y despertar?; ¿tuvo algún sueño durante el procedimiento? Estas preguntas deben ser realizadas idealmente en tres oportunidades, a saber, el post operatorio inmediato y luego a los 7 y 30 días post operatorio. La razón de ello es que más de $50 \%$ de los episodios de awareness no se pesquisan inmediatamente, sino que en evaluaciones posteriores ${ }^{13}$.

\section{Factores de riesgo para el desarrollo de awareness:}

Pueden ser divididos en tres grandes grupos:

a) Dependientes del paciente ${ }^{7,17}$ : Escasa reserva cardiovascular, antecedentes de awareness, uso crónico de drogas (especialmente recreacionales), edad (hasta 50 veces más frecuente en población pediátrica).

b) Dependientes del procedimiento ${ }^{12,18,19}$ : Cesárea con anestesia general, cirugía cardíaca, trauma.

c) Dependientes de la anestesia ${ }^{20,21}$ : Falla de equipo, intubación en secuencia rápida, anestesia superficial, como por ejemplo en situaciones de hipovolemia, sepsis, trauma entre otras, donde la condición hemodinámica o general del paciente no permite administrar un plano anestésico adecuado, uso de relajantes neuromusculares (considerado como uno de los principales factores de riesgo para desarrollar trastorno de estrés post traumático $)^{11,22}$.

\section{Clínica del awareness}

Durante la anestesia general, el paciente no es capaz de comunicarse verbalmente con el anestesiólogo, por lo que este debe basar sus decisiones en algunos signos clínicos que describimos anteriormente y en la monitorización de los signos vitales. Cuando no se obtiene el nivel de profundidad adecuado, el paciente puede presentar diversos grados de percepciones. La gran mayoría de los pacientes tienen percepciones auditivas, pudiendo en algunos casos relatar con absoluta claridad y certeza hechos que ocurrieron en el pabellón. En menor medida, los pacientes pueden presentar dolor o parálisis, generando mayor ansiedad y pánico, los que a su vez son los principales determinantes del desarrollo de trastorno de estrés post traumático y de fijación en la memoria ${ }^{22}$. 


\section{Consecuencias del awareness}

Los recuerdos de percepción intraoperatoria generan en un gran porcentaje de las personas un trauma psíquico lo suficientemente intenso como para el desarrollo de un trastorno de estrés post traumático (TEPT). Existen diversas cifras que van desde 30 hasta $70 \%$ de desarrollo de TEPT en los pacientes que presentan awareness según diversos estudios $^{13,22,23}$.

Un factor importante para que esto ocurra parece ser la expectativa puesta en la anestesia general. La mayoría de los adultos sabe que no deben tener recuerdos conscientes del procedimiento quirúrgico, en niños, en cambio, pese a existir awareness no existen reportes de TEPT como consecuencia de esto, lo que podría ser explicado por la falta de expectativas que tiene el niño con respecto a la anestesia general.

Existen diversas experiencias y percepciones al ocurrir awareness, algunas de estas parecen ser útiles como mecanismo de predicción de desarrollo de TEPT. En el estudio de Osterman ${ }^{23}$, en que encuentra una incidencia de 52\% de TEPT, detalla las experiencias intraoperatorias y post operatorias que experimentaron los pacientes. Dentro de las experiencias intraoperatorias, la más común fue la imposibilidad para comunicarse y que se acompaña de sensación de desamparo, terror, inseguridad, miedo y parálisis. Sin embargo, las experiencias de "haberse separado del cuerpo el algún momento" o sentir que en algún momento podían "escapar mentalmente" de lo que estaba ocurriendo fueron las experiencias que mejor podrían predecir el desarrollo de TEPT.

Estas experiencias que pueden clasificarse como disociativas ya han sido observadas en otros escenarios, como por ejemplo en soldados de guerra, en víctimas de accidentes automovilísticos y en sobrevivientes del Holocausto y han servido como factor de predicción de desarrollo de TEPT $^{24,25}$.

Al observar el detalle de las experiencias postoperatorias en el estudio de Osterman, los resultados obtenidos fueron que la sensación de inseguridad y el desamparo eran las sensaciones más comunes, seguidos por abandono y traición por parte del equipo médico y sentir que la imposibilidad de comunicarse persistía después de la operación ${ }^{23}$.

\section{Awareness y desarrollo de TEPT}

El desarrollo de TEPT, trastorno de ansiedad caracterizado por fenómenos de evitación, hipervigilancia y fenómenos intrusivos que surgen posterior a un evento traumático importante ${ }^{26}$, va a depender de las diversas respuestas que genera el trauma psíquico entre un individuo y otro y que están en función del significado que adquiera el hecho traumático (en este caso la percepción intraoperatoria), lo que dependerá a su vez de la interacción entre el tipo e intensidad del acontecimiento traumático, y también de la biografía, los factores biológicos y el contexto social del sujeto ${ }^{27}$. Algunos factores de riesgo para el desarrollo de TEPT se describen a continuación ${ }^{28}$.

También se han descrito factores protectores en el desarrollo de TEPT como son la capacidad de control que tiene el sujeto sobre la situación misma, la capacidad de predecir el hecho y la percepción de la amenaza ${ }^{29}$ (Tabla 1).

\section{Neurobiología del awareness}

El awareness, como hecho traumático importante, puede generar una disregulación en el sistema autonómico. En pacientes que desarrollan TEPT se ha visto que los índices de cortisol en el momento de producirse el trauma psíquico, son menores a los esperados, lo que genera un predominio del sistema nervioso simpático. Este

Tabla 1. Factores de riesgo para TEPT

\begin{tabular}{|ll|}
\hline Propios del sujeto & Relacionados al trauma \\
\hline Sexo femenino & Violaciones \\
\hline Introversión & Ataque sexual \\
$\begin{array}{l}\text { Antecedentes de T. } \\
\text { de Conducta }\end{array}$ & Awareness previo \\
\hline Soltero & Presenciar muertes, lesiones \\
\hline Edad media & Asaltos \\
\hline Bajo nivel educacional & Accidentes \\
\hline $\begin{array}{l}\text { Abuso de alcohol } \\
\text { Experiencia previa del } \\
\text { trauma }\end{array}$ & Experiencias de combate \\
Creencias personales & $\begin{array}{l}\text { Infarto al miocardio, AVE, } \\
\text { VIH }\end{array}$ \\
\hline
\end{tabular}


predominio del sistema nervioso simpático por sobre el parasimpático explicaría las alteraciones en el aprendizaje del evento, en el que los recuerdos del evento se codifican juntos a fenómenos de distrés, esto provocaría perpetuación de los síntomas y cambios biológicos secundarios ${ }^{30}$. También existe evidencia de mayor reactividad de receptores alfa 2 adrenérgicos, mayor reactividad de la amígdala y región paralímbica anterior frente a estímulos relacionados con el trauma, menor respuesta del cíngulo anterior y áreas orbitofrontales (generando efectos a largo plazo en la estructura y función cerebral) y disminución del volumen del hipocampo. Este hallazgo, además, podría indicar que pacientes con hipocampo disminuido antes del trauma podrían generar TEPT más fácil que aquellos con un tamaño del hipocampo normal $^{30,31}$.

\section{Epidemiología de TEPT en awareness}

Estudios norteamericanos estiman que la prevalencia de exposición a eventos traumáticos ocurren entre $39,1 \%$ y $60 \%$ a lo largo de la vida $^{32-34}$. Sin embargo, no todas las exposiciones a eventos traumáticos generan TEPT. Según los estudios de Kessler y Breslau, se estima que ante cualquier situación traumática entre $13,0 \%$ y $20,4 \%$ de las mujeres y entre $6,2 \%$ y $8,1 \%$ de los hombres desarrollará TEPT y que existe distinta prevalencia para el desarrollo de TEPT según los eventos traumáticos ${ }^{34-36}$. Al comparar este estudio con los de Osterman y Moerman ${ }^{22,23}$ observamos que el awareness es un hecho traumático que genera entre 50 y $70 \%$ TEPT, es decir, es un factor traumático que tiene mayor capacidad predictiva en comparación a desastres naturales, combates e incluso eventos traumáticos como violencia sexual. El estudio de van der Kolk ${ }^{37}$, también pone esto en evidencia. En su estudio, las situaciones que más provocan TEPT son el awareness con $56 \%$, violaciones $(48 \%)$ y el presenciar muertes o graves lesiones $(10,7 \%)$.

\section{Awareness, TEPT y comorbilidad}

La importancia del desarrollo de TEPT posterior al awareness la encontramos en sus comorbilidades. El TEPT es un cuadro que presenta alta comorbilidad (hasta $80 \%$ según algunos estudios) ${ }^{38,39}$. Kessler ${ }^{34}$ encontró que el TEPT se asociaba a otro diagnóstico en $17 \%$ de las mujeres y en $12 \%$ de los hombres, sin embargo, cuando estaba presente éste se asociaba con 3 o más diagnósticos en $44 \%$ de las mujeres y en 59\% de los hombres. Las cifras encontradas en este estudio muestran al TEPT asociado con abuso de alcohol en hombres $(51,9 \%)$, depresión mayor $(48 \%)$, trastornos de conducta en hombres $(43,3 \%)$, fobia simple $(30 \%)$, fobia social $(28 \%)$, abuso de alcohol en mujeres $(27,9 \%)$ y agorafobia en mujeres $(22,4 \%)^{34}$. Otros estudios también demuestran que el TEPT constituye en sí mismo un alto riesgo de intentos suicidas ${ }^{40}$.

Otras complicaciones médicas que puede generar el TEPT son las que se producen en situaciones relevantes como en el embarazo en el que se ha visto asociado a síntomas de parto prematuro, hiperémesis, crecimiento fetal excesivo, aborto espontáneo y embarazo ectópico ${ }^{41}$. Otra situación a destacar es el antecedente de maltrato severo en la infancia, el que se asocia a TEPT y a mayor riesgo de presentar tabaquismo, enfermedades de transmisión sexual, isquemia cardíaca, cáncer, accidentes vasculares cerebrales y diabetes, entre otras enfermedades ${ }^{42}$. En estudios realizados en mujeres víctimas de violación o asalto, se ha encontrado mayores indicadores de patología médica al compararlas con mujeres sin estos antecedentes ${ }^{43,44}$. Otro grupo estudiado son los veteranos de guerra, en el que se realizó un seguimiento por 20 años en aquellos que presentaban TEPT crónico y en el que se encontró una mayor prevalencia de enfermedades circulatorias, digestivas, músculo esqueléticas, del sistema nervioso, respiratorias e infecciosas en comparación con veteranos de guerra sin $\mathrm{TEPT}^{45}$.

\section{Awareness, TEPT y pronóstico}

Algunos factores importantes en el pronóstico de TEPT son el haber presentado un trauma previo severo, ser mujer y abusar de sustancias. La intensidad de los síntomas de TEPT fue el factor que determinó una peor evolución según los estudios de Zatzick ${ }^{46}$. Otros síntomas importantes que empeoran el pronóstico son la presencia de síntomas disociativos, que se encuentran al presentar el fenómeno de awareness según el estudio de Osterman ${ }^{23}$ y que, como señalamos anteriormente, se estudiaron también en víctimas de otros eventos vitales estresantes o traumáticos ${ }^{24,25}$. 
Aunque se sabe que gran parte de los pacientes se recuperan de un trauma psíquico sin intervención ${ }^{34}$, hay evidencia que sustenta la efectividad de la terapia cognitivo conductual en aquellos pacientes que presenten TEPT, especialmente en aquellos que presentan una alta sintomatología ${ }^{47}$.

\section{Conclusiones}

El fenómeno de awareness presenta una incidencia baja, pero considerando el gran número de procedimientos que se realizan bajo anestesia general, no podemos estar indiferentes ante su aparición. Tenemos la tarea de prevenir el fenómeno gracias a un manejo anestesiológico que debe incluir la detección de factores de riesgo para awareness y un manejo meticuloso de las drogas anestésicas durante la operación, quedando abierto la investigación y el desarrollo de técnicas que permitan objetivar mejor el grado de profundidad anestésica del paciente.

También es importante atender y acoger las inquietudes de los pacientes, ya sea en el pre y post operatorio para disminuir los grados de ansiedad que genera una intervención quirúrgica. La buena acogida y recepción de estas inquietudes y la escucha activa de las sensaciones que el paciente dice haber percibido durante la operación ayudaría a disminuir la sensación de abandono y traición que perciben los pacientes que sufren de awareness. Es importante considerar que los recuerdos de percepción puede ocurrir incluso meses después de la operación, por lo que un seguimiento mediante entrevistas o cuestionarios sería necesario en ciertas ocasiones, como por ejemplo en cirugía cardíaca, uso de relajantes musculares o en aquellos pacientes que ya han sufrido de awareness anteriormente.

Ante la detección de awareness debemos ofrecer apoyo psicológico, medida recomendada por la Joint Commission on Accreditation of Healthcare Organizations, para así poder identificar aquellos pacientes que sufran síntomas importantes de TEPT e intentar evitar sus complicaciones asociadas $^{48}$.

\section{Referencias}

1. Schacter DL. Implicit memory: History and current status. J Exp Psychol Learn Mem Cogn 1987; 13: 501-18.

2. Bonebakker AE, Jelicic M, Passchier J, Bonke B. Memory during general anesthesia: practical and methodological aspects. Conscious Cogn 1996; 5: 542-61.

3. Ghoneim MM. Awareness during anesthesia. Anesthesiology 2000; 92: 597-602.

4. Kalkman CJ, Drummond JC. Monitors of depth of anesthesia, quo vadis? Anesthesiology 2002; 96: 784-7.

5. Monk TG, Saini V, Weldon BC, Sigl JC. Anesthetic management and one-year mortality after noncardiac surgery. Anesth Analg 2005; 100: 4-10.

6. Guerra F. Awareness and recall. Int Anesthesiol Clin 1986; 24: 75-99.

7. Ghoneim MM, Block RI. Learning and consciousness during general anesthesia. Anesthesiology 1992; 76: 279305.

8. Osterman JE, van der Kolk BA. Awareness during anesthesia and posttraumatic stress disorder. Gen Hosp Psychiatry 1998; 20: 274-81.

9. Liu WH, Thorp TA, Graham SG, Aitkenhead AR. Incidence of awareness with recall during general anaesthesia. Anaesthesia 1991; 46: 435-7.

10. Sebel PS, Bowdle TA, Ghoneim MM, Rampil IJ, Padilla RE, Gan TJ, et al. The incidence of awareness during anesthesia: a multicenter United States study. Anesth Analg 2004; 99: 833-9.

11. Sandin RH, Enlund G, Samuelsson P, Lennmarken C. Awareness during anaesthesia: a prospective case study. Lancet 2000 26; 355: 707-11.

12. Bogetz MS, Katz JA. Recall of surgery for major trauma. Anesthesiology 1984; 61: 6-9.

13. Lennmarken C, Sydsjo G. Psychological consequences of awareness and their treatment. Best Pract Res Clin Anaesthesiol 2007; 21: 357-67.

14. Liu WH, Thorp TA, Graham SG, Aitkenhead AR. Incidence of awareness with recall during general anaesthesia. Anaesthesia 1991; 46: 435-7.

15. Heier T, Steen PA. Awareness in anaesthesia: incidence, consequences and prevention. Acta Anaesthesiol Scand 1996; 40: 1073-86.

16. Brice DD, Hetherington RR, Utting JE. A simple study of awareness and dreaming during anaesthesia. $\mathrm{Br} \mathrm{J}$ Anaesth 1970; 42: 535-42.

17. Davidson AJ, Huang GH, Czarnecki C, Gibson MA, Stewart SA, Jamsen K, et al. Awareness during anesthesia in children: a prospective cohort study. Anesth Analg 2005; 100: 653-61.

18. Lubke GH, Kerssens C, Gershon RY, Sebel PS. Memory formation during general anesthesia for emergency cesarean sections. Anesthesiology 2000; 92: 1029-34.

19. Lyons G, Macdonald R. Awareness during caesarean section. Anaesthesia 1991; 46: 62-4.

20. Ranta SO, Laurila R, Saario J, Ali-Melkkilä T, Hynynen 
M. Awareness with recall during general anesthesia: incidence and risk factors. Anesth Analg 1998; 86: 1084-9.

21. Dowd NP, Cheng DC, Karski JM, Wong DT, Munro JA, Sandler AN. Intraoperative awareness in fast-track cardiac anesthesia. Anesthesiology 1998; 89: 1068-73.

22. Moerman N, Bonke B, Oosting J. Awareness and recall during general anesthesia. Facts and feelings. Anesthesiology 1993; 79: 454-64.

23. Osterman, Hopper, Heran, Keane, van der Kolk. Awareness under anestesia and the development of posttraumatic stress disorder. Gen Hosp Psychiatry 2001; 23: 198-204.

24. Murray J, Ehlers A, Mayou RA. Dissociation and posttraumatic stress disorder: two prospectives studies od road traffic accident survivors. Br J Psychiatry 2002; 180: 363-8.

25. Halligan SL, Yehuda R. Assessing dissociation as risk factor for posttraumatic stress disorder: a study of adult offspring of Holocaust survivors. J Nerv Ment Dis 2002; 190: 429-36.

26. Diagnostic and statistical Manual of Mental Disorders. $4^{\text {rd }}$ ed, text revision. Washington, DC. American Psychiatric Association. American Psychiatric Press, 2000.

27. Ursano RJ, Kao T, Fullerton CS. PTSD and meaning: structuring human chaos. J Nerv Mental Dis 1992; 180: 756-9.

28. Testdone JE, Tarrier N. Posttraumatic stress disorder following medical illness and treatment. Clin Psychol Rev 2003; 23: 409-48.

29. Foa EB, Zinbarg R, Rothbaum BO. Uncontrollability and unpredictability in post-traumatic stress disorder: an animal model. Psychol Bull 1992; 112: 218-38.

30 Winter H, Irle E. Hippocampal volumen in adult burn patient with ant without post traumatic stress disorder. Am J Psychiatry 2004; 161: 2194-200.

31. Wignall EL, Dickson JM, Vaughan P, et al. Smaller hippocampal volumen in patients recent-oncet of post traumatic stress disorder. Biol Psychiatry 2004; 56: 8326.

32. Breslau N, Davis GC, Andreski P, Peterson E. Traumatic events and posttraumatic stress disorder in an urban population of young adults. Arch Gen Psychiatry 1991; 48: 216-22.

33. Davidson JR. Trauma: the impact of post-traumatic stress disorder. J Psychopharmacol 2000; 14 (2 Suppl 1): S5-12.

34. Kessler RC, Sonnega A, Bromet E, Hughes M, Nelson CB. Posttraumatic stress disorder in the National Comorbidity Survey. Arch Gen Psychiatry 1995; 52: 1048-60.
35. Breslau N, Chilcoat HD, Kessler RC, Peterson EL, Lucia VC. Vulnerability to assaultive violence: further specification of the sex difference in post-traumatic stress disorder. Psychol Med 1999; 29: 813-21.

36. Breslau N, Kessler R, Chilcoat HD, Schultz LR, Davis GC, Andreski P. Trauma and posttraumatic stress disorder in the community: the 1996 Detroit Area Survey of Trauma. Arch Gen Psychiatry 1998; 55; 626-32.

37. Van der Kolk B. Posttraumatic stress disorder and the nature of trauma. Dialogues Clin Neurosci 2000; 2: 7-22.

38. Solomon SD, Davidson JR. Trauma: prevalence, impairment, service use, and cost. J Clin Psychiatry 1997; 58 (suppl 9): 5-11.

39. Brady KT. Posttraumatic stress disorder and comorbidity: recognizing the many faces of PTSD. J Clin Psychiatry 1997; 58 (suppl 9): 12-5.

40. Ferrada-Noli M, Asberg M, Ormstad K, Lundin T, Sundbom E. Suicidal behavior after severe trauma. Part 1: PTSD diagnoses, psychiatric comorbidity, and assessment of suicidal bahavior. J Trauma Stress 1998; 11: 103-12.

41. Seng JS, Oakley DJ, Sampselle CM, Killion C, GrahamBermann S, Liberzon I. Posttraumatic stress disorder and pregnancy complications. Obstet Gynecol 2001; 97: 17-22.

42. Felitti VJ, Anda RF, Nordenberg D, Williamson DF, Spitz AM, Edwards V, et al. Relationship of childhood abuse and household dysfunction to many of the leading causes of death in adults: the adverse childhood experiences (ACE) study. Am J Prev Med 1998; 14: 245-58.

43. Koss MP, Koss PG, Woodroof WJ. Deleterious effects of criminal victimization on women's health and medical utilization. Arch Intern Med 1991; 151: 342-7.

44. Golding JM. Sexual assault history and physical health in randomly selected Los Angeles women. Health Psychol 1994; 13: 130-8.

45. Boscarino JA. Diseases among men 20 years after exposure to severe stress: implications for clinical research and medical care. Psychosom Med 1997; 59: 605-14.

46. Zatzick DF, Kang SM, Muller HG, Russo GE, Rivara FP, Katon W, et al. Predicting posttraumatic distress in hospitalized trauma survivors with acute injuries. Am J Psychiatry 2002; 159: 941-6.

47. Bisson JI, Cohen JA. Disseminating early interventions following trauma. J Trauma Stress 2006; 19: 583-95.

48. Joint Commission on Accreditation of Healthcare Organizations. Preventing and managing the impact of anesthesia awareness. Jt Comm Perspect 2004; 24: 10-1. 